\title{
DIFERENCIAS DENTRO DE LAS SALAS DE CLASES DISTRIBUCIÓN DEL RENDIMIENTO EN MATEMÁTICAS
}

\author{
María José Ramírez
}

\begin{abstract}
Este estudio analiza la distribución del rendimiento en matemáticas en alumnos de $4^{\circ}$ básico en Chile. Los resultados del SIMCE 1999 se analizaron en seis niveles: regiones, provincias, comunas, escuelas, sala de clases y alumnos dentro de las salas de clases. Los resultados indican que existen dos niveles relevantes de análisis: mientras cerca del 30\% de la varianza de puntajes se encuentra entre las escuelas, sobre el $70 \%$ se encuentra entre alumnos que asisten a la misma escuela. Una sala de clases típica cubre sobre tres desviaciones estándares de la distribución nacional de puntajes. Estos resultados cuestionan la supuesta homogeneidad de rendimiento dentro de las escuelas y el uso de una pedagogía que ignora los distintos niveles de rendimiento de los alumnos.
\end{abstract}

María José Ramírez. Ph. D. en educación, Boston College, EE.UU. Actualmente trabaja en la Universidad Diego Portales. 
$\amalg_{\text {n Chile, la discusión acerca de las diferencias de rendimiento aca- }}$ démico suele centrarse en la brecha existente entre distintas escuelas. Año a año, el SIMCE ${ }^{1}$ muestra las enormes diferencias entre las escuelas de distinto grupo socioeconómico (MINEDUC, 2005, 2006). Abundan los estudios que intentan explicar las diferencias de puntaje promedio entre las escuelas en función de su dependencia administrativa pública o privada (McEwan \& Carnoy, 2000; Mizala \& Romaguera, 2000), régimen de incentivos (Contreras et al., 2005) o la presencia de algún programa de intervención (Aylwin, 2003; García, 2006).

Sin embargo, pareciera no haber estudios a nivel nacional que analicen las diferencias de rendimiento dentro de las escuelas. Esto se debe, en parte, al supuesto implícito de que las diferencias de rendimiento se encuentran entre las escuelas, y no dentro de éstas. Este supuesto contrasta fuertemente con la evidencia internacional que muestra que es precisamente al interior de las escuelas donde se concentran las mayores diferencias de rendimiento.

Diversos autores han estudiado la estructura de la varianza de puntajes en pruebas estandarizadas para conocer la distribución del rendimiento académico de los alumnos de distintos países. La evaluación internacional TIMSS ha sido una fuente principal de información para estos estudios (Beaton \& O’Dwyer, 2002; Koretz, McCaffrey \& Sullivan, 2001; Martin et al., 2000; Stemler, 2001).

Stemler (2001) comparó la estructura de varianza de rendimiento de 14 países $^{2}$ y descompuso la distribución de puntajes de $4^{\circ}$ básico de matemáticas en dos niveles: varianza entre escuelas y varianza intraescuelas (que en rigor correspondía a la varianza intracursos). Él reporta que, en promedio, $70 \%$ de la varianza de puntajes corresponde a varianza intraescuelas. Sin embargo, los países muestran estructuras de varianza muy distintas entre sí. Por ejemplo, en Eslovenia la varianza entre escuelas es equivalente al $17 \%$ de la varianza total de puntajes. La otra cara de este resultado es que el $83 \%$ de la varianza de puntajes corresponde a varianza intraescuelas; es decir, corresponde a las diferencias de rendimiento entre compañeros de curso. Los resultados de Nueva Zelanda son más bien opuestos: $46 \%$ de la varianza de puntajes se encuentra entre las escuelas, sugiriendo que importantes diferencias existen en el puntaje promedio de las escuelas de este país.

${ }^{1}$ SIMCE es el sistema nacional de evaluación del Ministerio de Educación de Chile. Por ley, evalúa a todas las escuelas del país y publica sus resultados anualmente.

${ }^{2}$ Los 14 países analizados por Stemler (2001) son: Australia, Canadá, Chipre, Corea del Sur, Estados Unidos, Eslovenia, Grecia, Hong Kong, Irán, Irlanda, Letonia, Nueva Zelanda, Portugal y República Checa. 
En Estados Unidos, Beaton y O’Dwyer (2002) reportan que 35\% de la varianza de puntajes de matemáticas en $8^{\circ}$ básico se encuentra entre cursos de una misma escuela. Este resultado es reflejo de la práctica habitual de las escuelas norteamericanas de asignar a los alumnos a distintos cursos según su desempeño académico (tracking).

En Chile, la evidencia indica que hay importantes diferencias de rendimiento dentro de las escuelas, y que éstas van disminuyendo en los grados más avanzados. Así, mientras en $8^{\circ}$ básico el $61 \%$ de la varianza de puntajes se encuentra dentro de las escuelas (Ramírez, 2004), en $2^{\circ}$ medio ( $10^{\circ}$ grado) este porcentaje baja a 55\% (OECD-UIS, 2003, Anexo B7). Si bien estos resultados deben ser leídos con cautela, señalan un patrón necesario de tener en cuenta al estudiar la distribución de la varianza de puntajes en el país ${ }^{3}$.

Conocer dónde se concentran las diferencias de rendimiento entre los alumnos es importante para la política educacional. Si las mayores diferencias se concentran dentro de las escuelas, entonces es relevante preguntarse cómo las escuelas atienden a alumnos de rendimiento heterogéneo. Prácticas pedagógicas diseñadas para un grupo homogéneo de alumnos pueden no ser las más apropiadas si se tienen que implementar con cursos de rendimiento diverso. Por lo tanto, antes de diseñar una intervención destinada a mejorar el rendimiento académico de los alumnos es conveniente analizar la distribución de sus puntajes en pruebas estandarizadas.

Usando datos de la prueba SIMCE 1999 de matemáticas para $4^{\circ}$ básico, el objetivo de este estudio es analizar la varianza de puntajes de los alumnos en los seis niveles que permiten los datos: regiones, provincias, comunas, escuelas, cursos y alumnos dentro de los cursos. Las hipótesis que guiaron este estudio fueron:

- Las diferencias de rendimiento entre regiones y provincias son relativamente menores. Esto es esperable considerando que el Ministerio de Educación opera en forma centralizada a través de las Secretarías Regionales Ministeriales de Educación (Seremi) y de los Departamentos Provinciales de Educación (Deprov).

- Las diferencias de rendimiento entre comunas son de mediana magnitud. Esto es esperable considerando que los municipios son responsables de administrar las escuelas públicas de su comuna y que

${ }^{3}$ Los porcentajes de varianza intraescuela aquí reportados no son estrictamente comparables, ya que fueron obtenidos a partir de distintas pruebas (TIMSS en el caso de $8^{\circ}$ básico, PISA en el caso de $2^{\circ}$ medio) cuyos métodos de muestreo de alumnos también son diferentes (en cada escuela, TIMSS selecciona un curso entero de $8^{\circ}$ básico, mientras que PISA selecciona directamente alumnos de distintos cursos que pertenecen mayoritariamente a $2^{\circ}$ medio). 
los municipios presentan enormes diferencias en sus capacidades de gestión escolar (García-Huidobro, 2006, cap. IV).

- Las diferencias de rendimiento entre escuelas son de mayor magnitud en comparación con los 14 países estudiados por Stemler (2001). Esto es esperable por tres razones. Primero, porque el sistema escolar chileno está fuertemente estratificado por clases sociales y el nivel socioeconómico de los alumnos es un importante predictor del puntaje promedio de las escuelas (Mella, 2003; Mizala \& Romaguera, 2000; Ramírez, 2004, 2006a, 2006b). Segundo, por el efecto de las diferencias de gestión municipal explicado en el párrafo anterior. Tercero, por la mayor diversificación de la oferta (y calidad) educativa que es esperable en un sistema escolar con una fuerte participación de privados y con mínima regulación del Estado 4 .

- Las diferencias de rendimiento entre los cursos de las mismas escuelas son marginales. Esto porque en Chile las escuelas no suelen agrupar a sus alumnos en cursos de distinto rendimiento.

Este trabajo incluye una descripción de los datos y procedimientos utilizados para descomponer la varianza de puntajes de los alumnos. Los resultados muestran el porcentaje de la varianza de rendimiento que se encuentra a nivel de regiones, provincias, comunas, escuelas, cursos y alumnos dentro de los cursos. La última sección discute los resultados en un contexto nacional e internacional levantando temas de relevancia para la política educativa en Chile.

\section{Metodología}

El presente estudio usa datos de la prueba SIMCE 1999 de matemáticas. Esta prueba incluyó 45 preguntas distribuidas en dos cuadernillos de 30 preguntas cada uno (27 de selección múltiple y tres de desarrollo). Cada alumno contestó un cuadernillo y se le asignó un puntaje en función de sus respuestas. El SIMCE 1999 evaluó a 281.468 alumnos de $4^{\circ}$ básico pertenecientes a 5.470 escuelas (públicas y privadas). Estos alumnos representaban el 93\% del total de la matrícula de $4^{\circ}$ básico $^{5}$. El SIMCE 1999 estrenó una nueva escala de puntajes con una media $M=250$ puntos y desviación estándar $S=50$ (base de datos MINEDUC, 1999, 2000a, 2000b).

${ }^{4}$ El 42\% de las 5.470 escuelas evaluadas por SIMCE 1999 son privadas (particulares subvencionadas o pagadas).

${ }^{5} \mathrm{El} 7 \%$ restante de la población de alumnos de $4^{\circ}$ básico no fue evaluado en el SIMCE 1999 ya que pertenecía a escuelas muy pequeñas o geográficamente aisladas. 
La distribución de puntajes de los alumnos se descompuso utilizando análisis de la varianza unifactorial (one-way ANOVA). Primero, este análisis se hizo en forma independiente para las regiones, provincias, comunas, escuelas y cursos. En cada uno de estos niveles, $\eta^{2}$ (eta al cuadrado) se utilizó como indicador de la proporción de la varianza que se encuentra entre grupos (ej., regiones, escuelas). $\eta^{2}$ es la razón entre la suma de cuadrados entre grupos $\left(S C_{\text {entre }}\right)$ y la suma de cuadrados total $\left(S C_{\text {total }}\right)$ (ecuación 1).

$$
\eta^{2}=\frac{S C_{\text {entre }}}{S C_{\text {total }}}=\frac{\sum\left(\bar{\chi}_{. j}-\bar{\chi}_{.}\right)^{2}}{\sum\left(\chi_{i j}-\bar{\chi}_{.}\right)^{2}}
$$

Ecuación 1

En donde:

$\bar{\chi}_{. j}=$ media del grupo $j$

$\bar{\chi} . .=$ media nacional

$\chi_{i j}=$ puntaje del alumno $i$ en el grupo $j$

El objetivo del análisis era estimar la proporción de la varianza que se encuentra entre las unidades de un sistema (ej., provincias) después de descontar el efecto de las diferencias entre las unidades del nivel inmediatamente superior (ej., regiones). En otras palabras, el objetivo era estimar la proporción de la varianza de rendimiento que se encuentra entre las provincias de una misma región, para luego estimar la proporción de la varianza que se encuentra entre las comunas de una misma provincia, y así sucesivamente. Por ello, los resultados de ANOVA fueron ajustados para tomar en cuenta la estructura jerárquica de los niveles (ej., regiones que contienen provincias, provincias que contienen comunas). El ajuste se hizo descontando de cada nivel la proporción de la varianza entre grupos del nivel inmediatamente superior (ecuación 2).

$$
\eta_{\text {ajustado }}^{2}=\eta_{\text {nivel1 }}^{2}-\eta_{\text {nivel1+1 }}^{2}
$$

Ecuación 2

\section{Resultados}

La Tabla $\mathrm{N}^{\circ} 1$ muestra los resultados del análisis de la varianza de los puntajes de los alumnos en la prueba SIMCE 1999 de matemáticas a nivel de regiones, provincias, comunas, escuelas, cursos y alumnos dentro de los cursos. La penúltima columna muestra los resultados sin ajuste y la última 
TABLA N ${ }^{\circ}$ 1: $\quad$ DESCOMPOSICIÓN DE LA VARIANZA DE PUNTAJES DE LA PRUEBA SIMCE 1999 DE $4^{\circ}$ BÁSICO EN MATEMÁTICAS

\begin{tabular}{lrrrr}
\hline Nivel & $\begin{array}{c}\text { Grados de } \\
\text { libertad }\end{array}$ & $\begin{array}{c}\text { Suma de } \\
\text { cuadrados }\end{array}$ & $\begin{array}{c}\text { Proporción } \\
\text { de la varianza } \\
\text { entre grupos } \\
\left(\eta^{2}\right)\end{array}$ & $\begin{array}{c}\text { Proporción } \\
\text { de la varianza } \\
\text { ajustada } \\
\text { entre grupos } \\
\left(\eta^{2} \text { ajustado }\right)\end{array}$ \\
\hline Regiones & & & & \\
Provincias & 12 & 3.697 .455 & 0,005 & 0,005 \\
Comunas & 40 & 20.223 .031 & 0,029 & 0,024 \\
Escuelas & 333 & 44.595 .586 & 0,063 & 0,035 \\
Cursos & 5.469 & 191.000 .000 & 0,272 & 0,208 \\
Alumnos dentro de los cursos & 9.379 & 219.000 .000 & 0,312 & 0,040 \\
& 281.467 & 703.000 .000 & - & 0,688 \\
\hline
\end{tabular}

con ajuste. Tal como lo indica la última columna, el rendimiento promedio de los alumnos fue prácticamente el mismo de una región a otra. Sólo el 0,5\% del total de la varianza de rendimiento se explica por las diferencias de puntaje existentes entre las 13 regiones del país. A nivel provincial y comunal la proporción de la varianza entre grupos también es marginal (2,4\% y 3,5\% respectivamente).

Los resultados cambiaron drásticamente a nivel de escuelas. Sin el ajuste, las diferencias de puntaje promedio entre las escuelas dan cuenta del $27,2 \%$ de la varianza total de puntajes. Después de descontar el efecto de las diferencias entre regiones, provincias y comunas, las diferencias entre escuelas dan cuenta del 20,8\% de la varianza. De acuerdo con lo esperado, estos resultados confirman la existencia de importantes diferencias de rendimiento entre las escuelas chilenas.

En la fila de los cursos es interesante notar que, sin el ajuste, las diferencias entre ellos dan cuenta del 31,2\% de la varianza total de puntaje. Después del ajuste, este porcentaje baja a 4,0\%. Esta disminución se explica por la gran proporción de la varianza que se encuentra entre las escuelas y que no se considera nuevamente en el cálculo ajustado a nivel de cursos. Estos resultados indican que, dentro de una misma escuela, el puntaje promedio de los cursos tiende a ser prácticamente igual. Las diferencias de puntaje dentro de los cursos dan cuenta de la mayor proporción de la varianza de puntajes: 68,8\%. Estos resultados indican que los cursos están conformados por alumnos de rendimiento muy heterogéneo. El Gráfico $\mathrm{N}^{\mathrm{o}} 1$ resume los resultados de la tabla. 
GRÁFICO N ${ }^{\circ}$ 1: $\quad$ ESTRUCTURA DE LA VARIANZA DE PUNTAJES EN LA PRUEBA SIMCE 1999 DE $4^{\circ}$ BÁSICO EN MATEMÁTICAS*

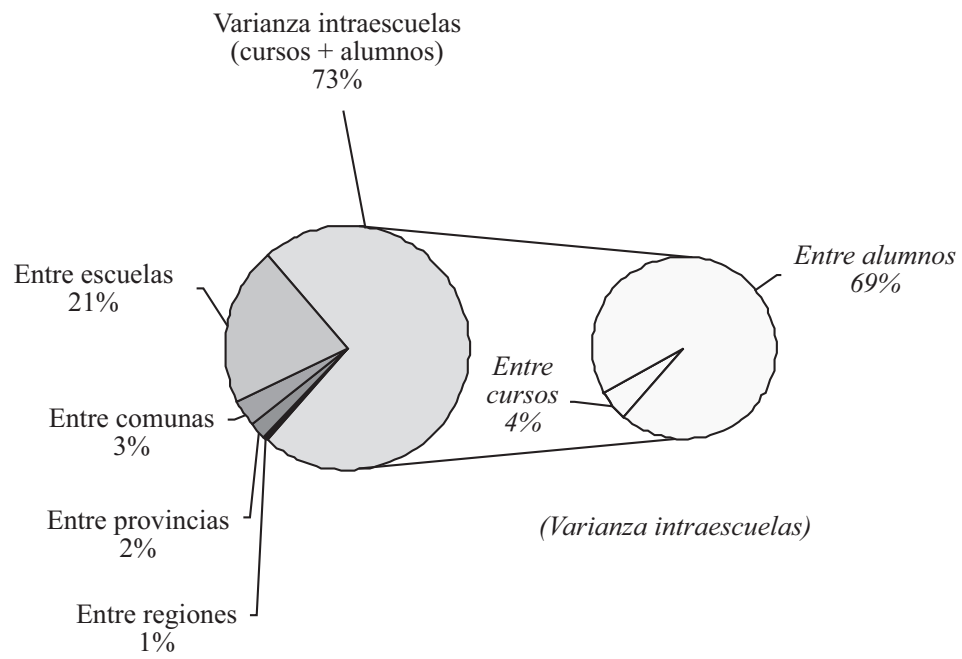

* Las áreas representan el porcentaje de la varianza total de puntajes que se encuentra en cada nivel. Los porcentajes asociados a cada nivel fueron ajustados para reflejar el efecto de las distintas unidades (ej., provincias) después de descontar el efecto del nivel inmediatamente superior (ej., regiones). Por ejemplo, el gráfico indica que $2 \%$ de la varianza total de puntajes se encuentra entre provincias de una misma región. Los porcentajes fueron redondeados al entero más cercano.

Considerando que las mayores diferencias de puntaje se concentran entre las escuelas y dentro de las salas de clases, se analizó en mayor detalle la dispersión de puntajes en estos dos niveles. El Gráfico $\mathrm{N}^{\circ} 2$ presenta la distribución de puntajes al interior de una muestra aleatoria de 150 escuelas. Es notable observar cómo las diferencias de puntaje entre las escuelas son relativamente menores comparadas con las diferencias de puntaje entre los alumnos de las mismas escuelas. También llama la atención que las escuelas con una mediana de puntajes relativamente baja tengan alumnos que logran muy alto rendimiento y que, por el contrario, escuelas con una mediana de puntajes relativamente alta tengan alumnos con muy bajo rendimiento. La distribución de puntajes en una escuela típica cubre un rango de 167 puntos, equivalentes a 3,35 desviaciones estándares de la distribución nacional de puntajes. Los alumnos de más bajo puntaje de cada escuela promedian 165 puntos, mientras que los de más alto puntaje promedian 332 puntos. En el primer caso se trata de alumnos que no alcanzan el 
GRÁFICO No 2: $\quad$ DISTRIBUCIÓN DE PUNTAJES DENTRO DE LAS ESCUELAS*

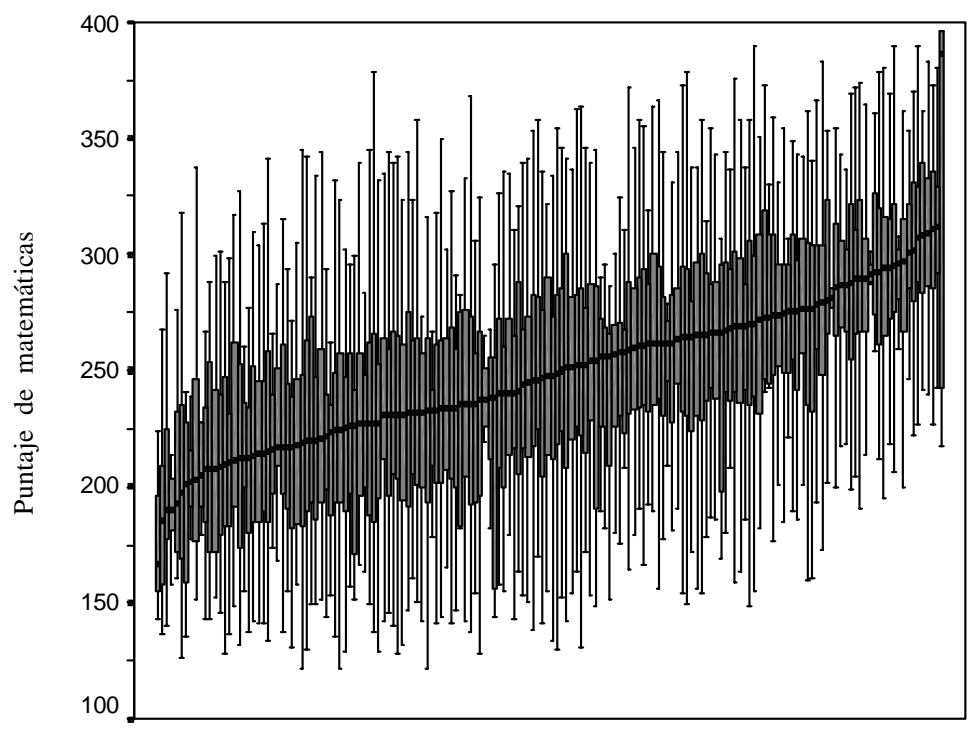

Escuelas

* El gráfico presenta la distribución de puntajes en la prueba SIMCE 1999 de matemáticas de una muestra aleatoria de 150 escuelas con 10 o más alumnos evaluados. Cada barrita representa una escuela. Las escuelas fueron ordenadas según su mediana (percentil 50 o puntaje que separa a la mitad de los alumnos de más alto rendimiento de la mitad de los alumnos de más bajo rendimiento). La zona gris muestra el rango de puntajes donde se concentra el 50\% de los alumnos de cada escuela (percentiles 25 y 75), las rectas indican los puntajes máximos y mínimos de cada escuela y los puntos los casos extremos. En Chile, una escuela típica cubre un rango de 167 puntos, equivalentes a 3,35 desviaciones estándares de la distribución nacional de puntajes. El promedio es de 54 alumnos de $4^{\circ}$ básico por escuela.

nivel básico de desempeño descrito en la prueba SIMCE 1999; en el segundo caso se trata de alumnos que alcanzan el nivel alto ${ }^{6}$.

El Gráfico $\mathrm{N}^{\circ} 3$ presenta la distribución de puntajes dentro de los 279 cursos de la misma muestra aleatoria de escuelas. El patrón es muy similar al gráfico anterior. La distribución de puntajes de un curso típico cubre un rango ligeramente menor: 161 puntos, equivalentes a 3,22 desviaciones estándares de la distribución nacional de puntajes. Los alumnos de más bajo

${ }^{6}$ El SIMCE 1999 describe cuatro niveles de desempeño (entre paréntesis se indica el porcentaje de alumnos en cada nivel): alto (12\%), intermedio (25\%), básico (31\%) y deficiente (32\%) (MINEDUC, 2000a). 


\section{GRÁFICO No 3: $\quad$ DISTRIBUCIÓN DE PUNTAJES DENTRO DE LOS CURSOS*}

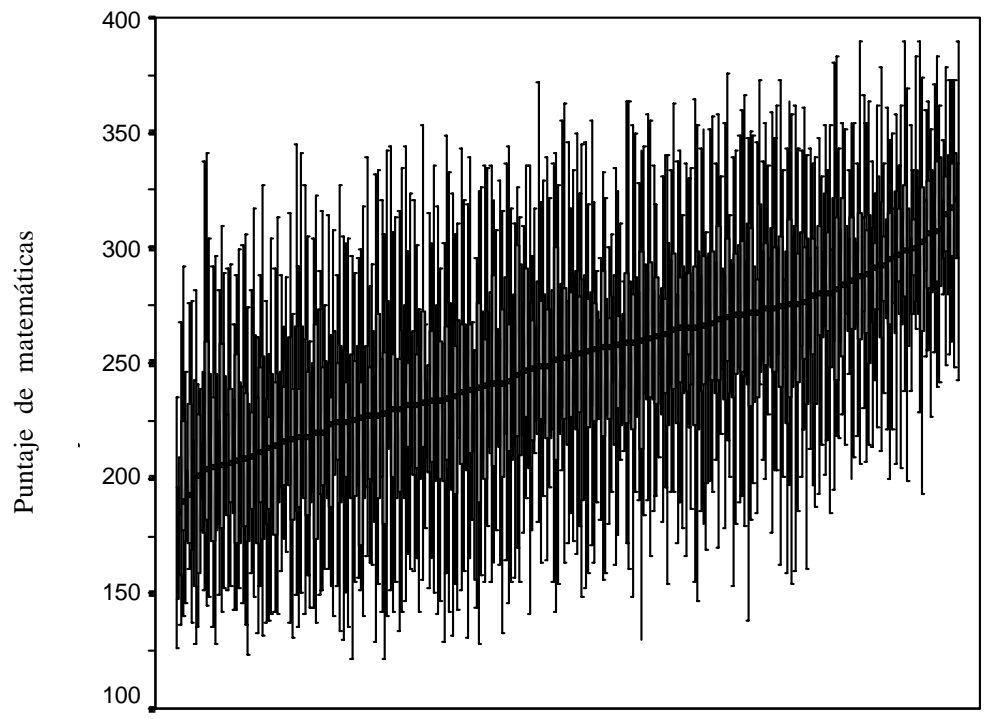

Cursos

* El gráfico presenta la distribución de puntajes en la prueba SIMCE 1999 de matemáticas de los 279 cursos de una muestra aleatoria de 150 escuelas con 10 o más alumnos evaluados. Cada barrita representa un curso. Los cursos fueron ordenados según su mediana (percentil 50 o puntaje que separa a la mitad de los alumnos de más alto rendimiento de la mitad de los alumnos de más bajo rendimiento). La zona gris muestra el rango de puntajes donde se concentra el $50 \%$ de los alumnos de cada curso (percentiles 25 y 75), las rectas indican los puntajes máximos y mínimos de cada curso y los puntos los casos extremos. En Chile, un curso típico cubre un rango de 161 puntos, equivalentes a 3,22 desviaciones estándares de la distribución nacional de puntajes. El promedio es de 32 alumnos por curso; $56 \%$ de las escuelas tiene un solo curso de $4^{\circ}$ básico.

puntaje de cada curso promedian 170 puntos, mientras que los de más alto puntaje promedian 331 puntos. Nuevamente, en el primer caso se trata de alumnos que no alcanzan el nivel de desempeño básico; en el segundo caso se trata de alumnos que alcanzan el nivel alto.

\section{Conclusiones e implicancias de política}

El análisis de la estructura de la varianza de puntajes de la prueba SIMCE 1999 de matemáticas muestra que, en términos generales, el rendimiento de los alumnos de $4^{\circ}$ básico se distribuye siguiendo un patrón 10 , 
20 , 70. Esto quiere decir que $10 \%$ de las diferencias de puntaje se encuentra distribuido a nivel de comunas, provincias y regiones; otro $20 \%$ corresponde a diferencias entre las escuelas de las mismas comunas, y el 70\% restante corresponde a diferencias de rendimiento dentro de las salas de clases.

Estos resultados confirman la hipótesis de que las diferencias de puntajes entre regiones y provincias son marginales. Sin embargo, ellos no confirman la hipótesis de que una parte importante de la varianza de rendimiento se encuentra entre las comunas. Tampoco sugieren que las escuelas chilenas difieren sustantivamente más entre sí que las escuelas de los 14 países analizados por Stemler (2001). En Chile, 31\% de la varianza de puntajes se encuentra entre cursos de distintas escuelas; en los 14 países analizados por Stemler, 30\%.

Los datos muestran que el $69 \%$ de la varianza de puntajes se encuentra dentro de las salas de clases, prácticamente lo mismo que reporta Stemler (2001) para sus 14 países. En Chile, un curso típico cubre sobre tres desviaciones estándares de la distribución nacional de puntajes. Lo usual es que dentro de un mismo curso haya desde alumnos que no alcanzan el nivel de desempeño básico de matemáticas hasta alumnos que alcanzan el nivel alto. De acuerdo con lo esperado, no se observan diferencias significativas de rendimiento entre los cursos de las mismas escuelas.

\section{Rompiendo mitos}

La estructura de distribución de la varianza de puntajes aquí reportada tiene importantes implicancias para la política educativa. El que las grandes diferencias de rendimiento se concentren en la sala de clases desmiente la idea de que los alumnos de bajo rendimiento están atrapados en escuelas mediocres. Los datos muestran que los alumnos con mayor retraso escolar se encuentran repartidos en todos los establecimientos educativos. Probablemente hay más de ellos en las escuelas grandes y no necesariamente en las escuelas con menor puntaje promedio (muchas de las cuales son pequeñas escuelas rurales).

Para asegurar que la mayor cantidad de alumnos de bajo rendimiento se beneficie con fondos o programas estatales, sería recomendable revisar la forma en que se focalizan recursos hacia las escuelas. Por ejemplo, podría considerarse la cantidad de alumnos de bajo rendimiento atendidos en cada escuela, y no sólo el puntaje promedio de ésta última (como usualmente ocurre). 
Estos resultados cuestionan la idea de que a una cierta edad y en un cierto grado los alumnos manejan un repertorio similar de contenidos y habilidades matemáticas. Los datos muestran que los cursos de $4^{\circ}$ básico están conformados por alumnos de muy diverso nivel de desempeño. Mientras algunos alumnos están listos para seguir progresando en el programa de estudios, otros aún están lidiando para aprender los contenidos ya pasados. Esta situación grafica las complejidades que presenta la labor docente. También impone un desafío enorme para el uso de estrategias pedagógicas efectivas.

\section{Cómo enseñar a alumnos de rendimiento heterogéneo}

En Chile, los profesores suelen tener conflictos éticos y conceptuales al momento de enfrentar el tema sobre cómo atender la diversidad de rendimientos entre alumnos de un mismo grado. El discurso dominante entre muchos es que lo justo es enseñarles a todos los alumnos por igual, sin diferenciar, sin discriminar. Lo curioso es que esta visión contrasta fuertemente con el discurso constructivista, también dominante en el profesorado, a saber, que hay que adecuar la pedagogía a las estructuras cognitivas del niño, a su nivel de comprensión y desarrollo. Vygotsky (1978) diría que el profesor debe trabajar en la "zona de desarrollo próximo” de sus alumnos. Lamentablemente, en la práctica, esta falta de reconocimiento de las diferencias individuales de rendimiento se traduce en un "enseñar para la media” que termina siendo muy poco efectivo para la mayoría de los alumnos.

La evidencia nacional e internacional es enfática en señalar que las escuelas efectivas se hacen cargo de las diferencias de rendimiento de sus alumnos. Después de revisar cientos de estudios de diversos países, Reynolds y Teddlie (2000) concluyen que un componente principal de la enseñanza de estas escuelas es la adaptación de las prácticas pedagógicas a las necesidades educativas de sus alumnos. Los profesores de las escuelas efectivas adaptan constantemente los programas de estudio y textos escolares según el nivel de sus alumnos, y evalúan a sus alumnos para saber qué tipo de atención requieren.

En Chile, Belleï, Muñoz, Pérez y Raczynski (2003) describen las escuelas efectivas así:

La atención de estas escuelas está puesta en lograr el aprendizaje de todos, y es por esto mismo que manejan la diversidad de alumnos y de los diferentes ritmos en que estos aprenden con estrategias —institucionales y pedagógicasespecialmente diseñadas para ello. La "cultura de la evalua- 
ción” que existe en estas escuelas se proyecta hacia la realización de diagnósticos sobre la situación personal y educativa de los alumnos. Estos diagnósticos facilitan que la escuela y sus profesores elaboren una planificación que se adapta a las potencialidades y limitaciones de los alumnos. Los alumnos con problemas o atrasos en su aprendizaje son tratados diferenciadamente, pero al mismo tiempo integrados en las actividades de la escuela. Esto es una constante, aunque se aborda de maneras distintas: proyectos de integración, apoyo especializado, trabajo de reforzamiento, guías de aprendizaje con distintos grados de dificultad, tutoría de pares. (P. 355.)

La evidencia internacional muestra diversas formas de organizar la enseñanza de alumnos de rendimiento heterogéneo. De $1^{\circ}$ a $6^{\circ}$ grado, lo usual es encontrar cursos muy heterogéneos. En Estados Unidos es común que el profesor presente un concepto, luego haga una práctica guiada y a continuación una práctica independiente dirigida al alumno promedio. Finalmente el profesor trabaja en grupos pequeños de acuerdo al nivel de comprensión de sus alumnos. Se forman tres grupos: uno que está en el nivel promedio, otro que está por debajo y otro que está por sobre el promedio. También existen programas de intervención (ej., cursos remediales) para aquellos alumnos que no pueden realizar las prácticas, pues no comprenden el concepto presentado por el profesor.

De $7^{\circ}$ a $12^{\circ}$ grado, la evidencia internacional muestra mayores niveles de diferenciación de los alumnos según su rendimiento. En Estados Unidos, Inglaterra y algunos sistemas escolares diseñados a partir del modelo inglés (ej., Hong Kong) es común que las escuelas agrupen a los alumnos en distintos cursos según su rendimiento académico. También es común la existencia de liceos de excelencia que sólo aceptan alumnos de alto rendimiento (al estilo de los liceos emblemáticos de Chile). La justificación para ello es que, al agrupar a los alumnos por rendimiento, el profesor puede adecuar mejor sus clases a las necesidades educativas de sus pupilos, maximizando así el aprendizaje tanto de los más rezagados como de los más avanzados.

Hay evidencia tanto a favor como en contra de la formación de cursos de rendimiento homogéneo. Algunos estudios indican que los alumnos se ven favorecidos al pertenecer a cursos de mejor rendimiento (Hoxby, 2002). Otros estudios muestran que los alumnos se ven favorecidos al pertenecer a cursos con alumnos de similares características, incluso si se trata de alumnos de bajo rendimiento (Hoxby \& Weingarth, 2005). También hay evidencia que indica que mientras los alumnos de alto rendimiento se ven 
favorecidos al pertenecer a cursos de mejor desempeño, los alumnos de bajo rendimiento se ven desfavorecidos al pertenecer a cursos de bajo desempeño (Ryoo, 2001). En Estados Unidos la evidencia muestra que los cursos de menor rendimiento suelen tener a los peores profesores, y que éstos tienen tan bajas expectativas respecto a sus alumnos, que el aprendizaje, lejos de potenciarse, se ve limitado (Hallinan, 2004; Oakes, 2005).

Considerando que el sistema escolar chileno está conformado por cursos heterogéneos, una recomendación de política importante es enseñar a los futuros profesores cómo trabajar con alumnos de diverso rendimiento. Lamentablemente, cuando las facultades de educación abordan el tema de las diferencias individuales suelen hacerlo desde el punto de vista de los trastornos de aprendizaje o desde un punto de vista culturalista; no abordan el desafío más masivo y complejo, cual es que los alumnos tienen distintos niveles de desempeño y ritmos de aprendizaje.

¿Qué explica las diferencias de rendimiento dentro de las salas de clases?

Diversos estudios muestran que el nivel socioeconómico de los alumnos es un fuerte predictor del rendimiento promedio de las escuelas. Sin embargo, su efecto es neutralizado cuando se analiza dentro de la sala de clases:

...una vez que los alumnos son asignados a un curso de matemáticas, el estatus socioeconómico deja de ser un predictor importante de su competencia en matemáticas. Dicho de otra manera, una vez que los alumnos han ingresado a un curso de matemáticas, tener padres con estudios superiores o una computadora en el hogar tiene poco que ver con el rendimiento en la asignatura. El estatus socioeconómico tal vez haya tenido un rol destacado en la asignación de los alumnos a un curso, pero no lo tiene en los resultados dentro de la sala de clases. (Beaton \& O’Dwyer, 2002, p. 229.)

El que las grandes diferencias de rendimiento se encuentren entre compañeros de curso que provienen de condiciones económicas y sociales similares cuestiona los determinismos sociales del tipo "a los ricos les va bien y a los pobres les va mal”. Miles de niños pobres del país se ubican del lado de los mejores puntajes de la distribución de desempeños. De igual forma, miles de niños de los estratos más acomodados muestran resultados bastante precarios. Con esto no se quiere obviar el enorme impacto que tienen en el rendimiento escolar variables como el ingreso familiar y la edu- 
cación de los padres. Sin embargo, estas variables dan cuenta de las diferencias de rendimiento entre las escuelas, no dentro de las escuelas. En la sala de clases, son otras las variables en juego.

Ramírez $(2004,2005)$ modeló el rendimiento en matemáticas de alumnos chilenos de $8^{\circ}$ básico. Sus resultados confirman que, en la sala de clases, el nivel socioeconómico de los alumnos no es un predictor significativo de rendimiento escolar. Esto se debe, en parte, a que el nivel socioeconómico es neutralizado en las salas de clases, y en parte a que el nivel socioeconómico de los alumnos es más homogéneo dentro de cada curso y escuela. Sí son relevantes para comprender las diferencias de rendimiento en las salas de clases las creencias y actitudes de los alumnos. Los alumnos que esperan realizar estudios universitarios y que atribuyen menos importancia a la suerte o inteligencia innata para aprender matemáticas obtienen sistemáticamente mejores resultados que sus compañeros con menores expectativas de estudio y que atribuyen más importancia a la suerte o inteligencia innata. Estos resultados sugieren que es importante desarrollar una cultura escolar en la cual el desempeño de los alumnos se perciba más dependiente del propio esfuerzo y motivación, y menos dependiente de factores no controlables por los alumnos.

\section{Equidad y justicia en educación}

Los resultados de este estudio llevan a preguntarse cuál es la distribución ideal de puntajes en un sistema escolar. ¿Es razonable esperar que no existan diferencias de rendimiento entre los alumnos? En todos los países de los que se tiene evidencia empírica existen grandes diferencias de rendimiento entre sus alumnos. Este patrón se observa incluso en los países que aparecen primeros en los rankings internacionales (Mullis et al., 2000, 2004; OECD-UIS, 2003). Por lo tanto, no parece razonable pretender eliminar las diferencias de desempeño entre los alumnos chilenos.

$¿$ Es razonable esperar que no existan diferencias de rendimiento entre las escuelas? La evidencia internacional indica que esto sí es posible. Corea del Sur es un caso interesante en este sentido. Este país es uno de los primeros en los rankings internacionales $\mathrm{y}$, al mismo tiempo, todas sus escuelas presentan un rendimiento similar entre ellas. De hecho, la varianza total de puntajes entre los cursos de distintas escuelas coreanas gira en torno al 5\% (versus 31\% en Chile). La fórmula de política para minimizar las diferencias de rendimiento entre las escuelas ha sido que las mismas reglas del juego corren para las escuelas públicas y las privadas. Por ejemplo, los alumnos coreanos son asignados por sorteo a una escuela (ya sea pública o 
privada subvencionada) de su zona residencial, y los profesores rotan de escuela cada cuatro años (Park \& Park, 2006).

Considerando que la elección de escuelas por parte de los padres está muy asentada en nuestra cultura nacional, pareciera poco viable eliminar este derecho en Chile. Sin embargo, se podrían implementar otras medidas para disminuir las diferencias de rendimiento entre las escuelas. Por ejemplo, asignando incentivos monetarios a los mejores egresados de pedagogía para que trabajen en escuelas de bajo rendimiento promedio. También se podría regular la selección académica de los alumnos, de modo tal que ésta sea similar (en cantidad y calidad) en todas las escuelas subvencionadas (públicas y privadas). Finalmente, se podrían fomentar aún más las políticas de discriminación positiva que entregan mayores recursos a las escuelas que atienden a los alumnos más pobres.

Cuando las diferencias de rendimiento son mínimas entre las escuelas, se puede afirmar con mayor certeza que todos los alumnos tienen similares oportunidades de aprendizaje, independientemente de la escuela a la que asisten. Cuando todos los alumnos tienen similares oportunidades de aprendizaje, las diferencias de rendimiento dentro de las escuelas pueden entenderse como más dependientes de las características de cada alumno, tales como su esfuerzo y su motivación por aprender (ver Stemler, 2001).

Los países con altos estándares de calidad y equidad en educación también se caracterizan porque sus alumnos de más bajo rendimiento alcanzan niveles suficientes para seguir avanzando exitosamente en la escuela. Chile tiene un desafío pendiente a este respecto, ya que un tercio de sus alumnos no alcanza este nivel mínimo (MINEDUC, 2000a). Mejorar los cursos remediales ya existentes en muchas escuelas del país puede ser parte de la solución a este problema ${ }^{7}$.

Además, Chile tiene un desafío pendiente en cuanto a dar más y mejores oportunidades de aprendizaje a sus alumnos de alto rendimiento. En un sistema escolar de calidad, estos alumnos también deberían tener clases estimulantes que les permitan maximizar su potencial de aprendizaje. No parece justo que a los alumnos más avanzados se les deje de pasar la materia que estipula el currículo nacional porque tienen compañeros de curso que todavía no están listos para dar ese paso. Cuando los profesores pasan más materia (es decir, cuando dan mejores oportunidades de aprendizaje) sus alumnos alcanzan mejor rendimiento promedio que cuando pasan menos materia (Gau, 1997; Ramírez, 2004, 2006b; Secada, 1992).

${ }^{7}$ El 83\% de los alumnos de $8^{\circ}$ básico asiste a una escuela que ofrece cursos remediales de matemáticas (Ramírez, 2004). 
Este estudio pretende contribuir a tomar conciencia sobre las grandes diferencias de rendimiento que existen entre compañeros de curso que asisten a la misma escuela y sala de clases. También pretende contribuir al debate sobre cuál es la forma más justa y eficaz de enseñar a alumnos de rendimiento heterogéneo. Queda pendiente el estudio de la estructura de varianza de puntajes en otras disciplinas (ej., lenguaje, ciencias) y grados ( $8^{\circ}$ básico, $2^{\circ}$ medio).

\section{REFERENCIAS}

Aylwin, M. (2003): “Los Programas Focalizados”. En R. Hevia (ed.), La Educación en Chile, Hoy (pp. 151-164). Santiago, Chile: Ediciones Universidad Diego Portales.

Beaton, A. E., \& L. M. O’Dwyer (2002): “Separating School, Classroom, and Student Variances and their Relationship to Socio-Economic Status". En D. F. Robitaille y A. E. Beaton (eds.), Secondary Analysis of the TIMSS Data (pp. 211-231). Boston: Kluwer Academic Publishers.

Belleï, C., G. Muñoz, L. M. Pérez y D. Raczynski (2003): "Escuelas Efectivas en Sectores de Pobreza, ¿Quién Dijo que no se Puede?”. En R. Hevia (ed.), La Educación en Chile, Hoy (pp. 347-372). Santiago de Chile: Ediciones Universidad Diego Portales.

Contreras, D., L. Flores, F. Lobato \& V. Macías (2005, marzo): "Monetary Incentives for Teachers and School Performance: Evidence for Chile”. Presentado en Research for Results in Education: A Global Conference on Education Research in Developing Countries, Praga, República Checa.

García, A. (2006, septiembre): "Evaluación del Impacto de la Jornada Escolar Completa”. Artículo presentado en el Encuentro de la Sociedad de Economía de Chile. La Serena, Chile. Descargado en enero 2007 desde http://sechi.facea.uchile.cl/sechi/ afiches/garcia_alva2.pdf

García-Huidobro, J. E. (2006). Informe Final del Consejo Asesor Presidencial para la Calidad de la Educación. Ministerio de Educación, Chile.

Gau, S. (1997, marzo): "The Distribution and the Effects of Opportunity to Learn on Mathematics Achievement”. Presentado en la reunión anual de la American Educational Research Association, Chicago, IL. (ERIC Document Reproduction Service $N^{\circ}$ ED407231.)

Hallinan, M. (2004): “The Detracking Movement”. En Education Next, otoño 2004. Descargado en enero 2007 desde http://media.hoover.org/documents/ ednext20044_72.pdf

Hoxby, C. (2002): “The Power of Peers: How Does the Makeup of Classroom Influence Achievement?” En Education Next, verano 2002. Descargado en enero 2007 desde http://media.hoover.org/documents/ednext20022_56.pdf

Hoxby, C. \& G. Weingarth (2005): “Taking Race Out of the Equation: School Reassignment and the Structure of Peer Effects”. Downloaded February 2007 from http://www.economics.harvard.edu/faculty/hoxby/papers/hoxby_weingarth_ taking_race.pdf 
Koretz, D., D. McCaffrey \& T. Sullivan (2001): "Predicting Variations in Mathematics Performance in Four Countries Using TIMSS”. En Educational Policy Archives, 9 (34). Descargado en enero 2007 desde http://epaa.asu.edu/epaa/v9n34/

Martin, M. O., I. Mullis, K. Gregory, C. Hoyle \& C. Shen (2000): Effective Schools in Science and Mathematics IEA's Third International Mathematics and Science Study. Chestnut Hill, MA: International Study Center, Lynch School of Education, Boston College.

McEwan, P. J. \& M. Carnoy (2000): "The Effectiveness and Efficiency of Private Schools in Chile's Voucher System”. En Educational Evaluation and Policy Analysis, 22 (3), pp. 213-239.

Mella, O. (2003): "12 Años de Reforma Educacional en Chile: Algunas Consideraciones en torno a sus Efectos para Reducir la Inequidad”. En Revista Electrónica Iberoamericana sobre Calidad, Eficacia y Cambio en Educación. Descargado en enero 2007 desde http://www.redalyc.uaemex.mx/redalyc/pdf/551/55110102.pdf

MINEDUC (Ministerio de Educación, Chile) (1999): Base de Datos SIMCE 1999 [archivo electrónico]. Santiago, Chile: Ministerio de Educación.

(2000a): Informe de Resultados SIMCE 1999 Cuarto Año de Educación Básica. Santiago, Chile: Ministerio de Educación, Unidad de Curriculum y Evaluación. Descargado en enero 2007 desde http://www.simce.cl/doc/ informe_1999_4basico.pdf (2000b): Informe Técnico Prueba SIMCE 1999 Cuarto Básico. Santiago, Chile: Ministerio de Educación, Unidad de Curriculum y Evaluación.

(2005): Informe de Resultados $8^{\circ}$ Básico SIMCE 2004. Descargado en enero 2007 desde http://www.simce.cl/doc/informe_resultados_2004.pdf

- (2006). Informe de Resultados $4^{\circ}$ Básico SIMCE 2005. Descargado en enero 2007 desde http://www.simce.cl/doc/simce2005_informe_resultados.pdf

Mizala, A. \& P. Romaguera (2000): "School Performance and Choice”. En Journal of Human Resources, 35 (2), 392-417.

Mullis, I. V. S., M. O. Martin, E. J. Gonzalez, K. D. Gregory, R. A. Garden, K. A. O’Connor, S. J. Chrostowski \& T. A. Smith (2000): TIMSS 1999 International Mathematics Report: Findings from IEA's Repeat of the Third International Mathematics and Science Study at the Eighth Grade. Chestnut Hill, MA: International Study Center, Lynch School of Education, Boston College.

Mullis, I. V. S., M. O. Martin, E. J. Gonzalez, S. J. Chrostowski (2004): TIMSS 2003 International Mathematics Report: Findings from IEA's Trends in International Mathematics and Science Study at the Fourth and Eighth Grades. Chestnut Hill, MA: International Study Center, Lynch School of Education, Boston College.

Oackes, J. (2005): Keeping Track: How Schools Structure Inequality (segunda edición). New Haven: Yale University Press.

OECD-UIS (Organization for Economic Cooperation and Development \& Unesco Institute for Statistics) (2003): Literacy Skills for the World of Tomorrow. Further Results from PISA 2000. Paris: OECD.

Park, C., \& D. Park (2006): "Factors Affecting Korean Students' Achievement in TIMSS 1999”. En S. J. Howie \& T. Plomp (eds.), Context of Learning Mathematics and Science: Lessons Learned from TIMSS. The Netherlands: Routledge Falmer. 
Ramírez, M. J. (2004): "Understanding the Low Mathematics Achievement of Chilean Students: A Cross-National Analysis Using TIMSS Data”. Doctoral dissertation, Boston College. Descargado en enero 2007 desde http://www.eric.ed.gov/ ERICWebPortal/Home.portal?_nfpb=true\&ERICExtSearch_SearchValue_0= understanding+the+low+mathematics+achievement+of+chilean\&ERICExtSearch_ SearchType_0=ti\&_pageLabel=RecordDetails\&objectId=0900000b8067af23\&accno $=$ ED491679

(2005): "Attitudes Toward Mathematics and Academic Performance among Chilean Eighth Graders”. En Estudios Pedagógicos, 31, pp. 97-112. Universidad Austral, Valdivia, Chile. Descargado en enero 2007 desde: http://www.scielo.cl/ scielo.php?pid=S0718-07052005000100006\&script=sci_arttext\&tlng=en

(2006a): "Factors Related to Mathematics Achievement in Chile". En S. J. Howie \& T. Plomp (eds.), Context of Learning Mathematics and Science: Lessons Learned from TIMSS (pp. 97-111). London: Routledge Taylor \& Francis Group.

(2006b): "Understanding the Low Mathematics Achievement of Chilean Students: A Cross-National Analysis Using TIMSS Data”. En International Journal of Educational Research, 45, 102-116.

Reynolds, D., \& C. Teddlie (2000): “The Processes of School Effectiveness”. En C. Teddlie \& D. Reynolds (eds.), The International Handbook of School Effectiveness Research (pp. 134-159). London: Falmer Press.

Ryoo, H. S. (2001): "Multilevel Influences on Student Achievement: An International Comparative Study”. Doctoral dissertation, Cornell University, 2001. Dissertation Abstracts International, 62 (03A).

Secada, W. (1992): "Race, Ethnicity, Social Class, Language, and Achievement in Mathematics”. En D. A. Grouws (ed.), Handbook of Research on Mathematics Teaching and Learning: A Project of the National Council of Teachers of Mathematics (pp. 623-660). New York: MacMillan Publishing Company.

Stemler, S. E. (2001): "Examining School Effectiveness at the Fourth Grade: A Hierarchical Analysis of the Third International Mathematics and Science Study (TIMSS)”. Doctoral dissertation, Boston College, 2001. Dissertation Abstracts International, 62, 03A.

Vygotsky, L. S. (1978): Mind in Society: The Development of Higher Psychological Processes. Cambridge, MA: Harvard University Press. 